\title{
Endothelial dysfunction and immunothrombosis as key pathogenic mechanisms in COVID-19
}

\section{Aldo Bonaventura (D), Alessandra Vecchié, Lorenzo Dagna, Kimberly Martinod(D, Dave L. Dixon, Benjamin W. Van Tassell, Francesco Dentali, Fabrizio Montecucco (D), Steffen Massberg (D), Marcel Levi and Antonio Abbate}

Abstract | Coronavirus disease 2019 (COVID-19) is a clinical syndrome caused by infection with severe acute respiratory syndrome coronavirus 2 (SARS-CoV-2). Patients with severe disease show hyperactivation of the immune system, which can affect multiple organs besides the lungs. Here, we propose that SARS-CoV-2 infection induces a process known as immunothrombosis, in which activated neutrophils and monocytes interact with platelets and the coagulation cascade, leading to intravascular clot formation in small and larger vessels. Microthrombotic complications may contribute to acute respiratory distress syndrome (ARDS) and other organ dysfunctions. Therapeutic strategies aimed at reducing immunothrombosis may therefore be useful. Several antithrombotic and immunomodulating drugs have been proposed as candidates to treat patients with SARS-CoV-2 infection. The growing understanding of SARS-CoV-2 infection pathogenesis and how it contributes to critical illness and its complications may help to improve risk stratification and develop targeted therapies to reduce the acute and long-term consequences of this disease.

Coronavirus disease 2019 (COVID-19)

is caused by the severe acute respiratory syndrome coronavirus 2 (SARS-CoV-2) and can present with a range of manifestations, including pneumonia and acute respiratory distress syndrome (ARDS) ${ }^{1}$. Although many individuals develop asymptomatic or paucisymptomatic SARS-CoV-2 infection, those with severe-to-critical disease show a dysregulated hyperactivation of the immune system that can cause an abnormal cytokine immune response and is likely to be responsible for most of the organ damage occurring in the late phase of the disease ${ }^{2}$.

It is now apparent that SARS-CoV-2 infection induces a prothrombotic state manifesting especially with microthrombosis ${ }^{3,4}$, as observed in patients with COVID-19 and in post-mortem samples from individuals who died after SARS-CoV-2 infection ${ }^{5-9}$. This thrombotic state is reminiscent of a process known as immunothrombosis (BOX 1; FIG. 1), in which the immune and coagulation systems cooperate to block pathogens and limit their spread ${ }^{10}$. In this Perspective, we describe how SARS-CoV-2 might induce a prothrombotic state and discuss how currently available and developing therapies may address COVID-19-associated immunothrombosis.

\section{Immunothrombosis in COVID-19} COVID-19-associated hyperinflammation. The clinical spectrum of COVID-19 ranges from asymptomatic or paucisymptomatic (in up to $45 \%$ of individuals) to severe disease requiring admission of patients to the intensive care unit (ICU) ${ }^{11}$. Generally, $3-10 \%$ of subjects requires hospitalization, with up to $20 \%$ of them experiencing severe disease and a high mortality rate $\mathrm{rat}^{12,13}$. The initial phase of COVID-19 is characterized by damage directly driven by the virus. After approximately 7-10 days of symptoms (including fever, cough, fatigue, anorexia and myalgias), a subgroup of patients progresses to severe disease and develops hypoxaemia (low oxygen levels in the blood) and dyspnoea (shortness of breath), potentially evolving towards ARDS ${ }^{14}$. This stage of the disease is characterized by high levels of pro-inflammatory cytokines, such as interleukin-6 (IL-6), IL-1 $\beta$, IL-18 and granulocyte-macrophage colony-stimulating factor (GM-CSF) $)^{2,15-17}$. This event has been referred to as a cytokine storm ${ }^{18,19}$, although recent evidence suggests that cytokine levels in patients with COVID-19 are lower than those reported in patients with non-COVID-19 ARDS, sepsis, influenza virus infection and chimeric antigen receptor (CAR) T cell-induced cytokine release syndrome ${ }^{20,21}$. SARS-CoV-2 has been shown to trigger the IL-1/IL-6 pathway to a larger extent than other coronaviruses ${ }^{22}$. Through the analysis of lungs of patients who died from COVID-19-related ARDS, SARS-CoV-2 was found to activate the NLRP3 inflammasome ${ }^{23,24}$. In vitro studies also showed NLRP3 inflammasome activation by SARS-CoV-2 infection of primary human monocytes. In addition, NLRP3 inflammasome products, such as IL- $1 \beta$ and IL-18, are increased in patients with severe COVID-19 and positively correlated with adverse clinical outcomes ${ }^{24}$. These findings support a potential relationship between SARS-CoV-2 infection and inflammatory features, such as the dysregulated cytokine response.

Increased levels of ferritin have also been observed among patients with COVID-19, similar to what is observed in macrophage activation syndrome ${ }^{16,19,25}$. Even though ferritin levels observed in macrophage activation syndrome are markedly higher than those currently observed in COVID-19 $(\geq 10,000$ $100,000 \mathrm{ng} \mathrm{ml}^{-1}$ versus $500-3,000 \mathrm{ng} \mathrm{ml}^{-1}$ ), ferritin levels remain predictive of poor outcomes in COVID-19 (REFS ${ }^{26,27}$ ).

With regard to inflammatory cells, large numbers of $\mathrm{CD} 8^{+}$macrophages were observed in the alveoli of patients who died from COVID-19-associated pneumonia, and SARS-CoV-2 proteins were detected in $\mathrm{CD}^{+} 8^{+}$alveolar macrophages ${ }^{28,29}$. Analysis of the bronchoalveolar fluid of patients with severe or critical COVID-19 showed a higher percentage of macrophages and neutrophils and a lower proportion of myeloid dendritic cells, plasmacytoid 
dendritic cells and T cells than that of patients with moderate disease ${ }^{30}$. They also had higher levels of pro-inflammatory cytokines (such as IL-6, IL-8 and IL-1 $\beta$ ) and chemokines (such as CCL2, CCL3, CCL4 and CCL7) in the bronchoalveolar fluid ${ }^{30}$. These data suggest that lung macrophages contribute to inflammatory responses in SARS-CoV-2 infection, favouring excessive inflammation in severe pneumonia. Similar to other respiratory infections, leukocyte recruitment to the lungs is finely tuned by specific molecules ${ }^{31}$, the uncontrolled activity of which may result in complications observed in patients with COVID-19 in both the lungs and other organs ${ }^{5,32}$.

COVID-19-associated coagulopathy. An important pathophysiological feature of COVID-19 is the development of a prothrombotic state. The occurrence of a COVID-19-specific coagulopathy is suggested by elevated levels of fibrinogen, von Willebrand factor (VWF) and the fibrin degradation product $\mathrm{D}$-dimer in the blood, whereas patients generally show minor or no changes in prothrombin time (a measure of time to clot), activated partial thromboplastin time (coagulation time), antithrombin levels, activated protein C levels and platelet count ${ }^{33-36}$.
These features are more consistent with a state of hypercoagulability coupled with a severe inflammatory state rather than with classical disseminated intravascular coagulation $(\mathrm{DIC})^{36}$. Indeed, DIC has been reported in a few patients, usually in the advanced stages of the disease $\mathrm{s}^{33,37,38}$. Recently, low antithrombin levels were linked to poor outcomes in COVID-19, especially among patients with obesity ${ }^{39}$. Hence, it is possible that antithrombin activity changes as SARS-CoV-2 infection worsens and a putative mechanism might be the cytokine-driven imbalance in endogenous anticoagulant levels ${ }^{40}$. In macrophage activation syndrome, liver function impairment is a typical feature and may contribute to coagulopathy, yet liver impairment in COVID-19 is usually mild and transient, without overt acute liver injury ${ }^{41,42}$.

Apart from a state of hypercoagulability in the lungs of patients with ARDS, a hypofibrinolytic state in the alveolar space was observed in COVID-19-related ARDS due to increased levels of fibrinolytic inhibitors $^{43}$. Accordingly, high levels of plasminogen activator inhibitor 1 (PAI1) were described in patients with SARS-CoV ${ }^{44}$.

In order to properly identify COVID19-associated coagulopathy, Iba et al.

\section{Box 1 | Immunothrombosis: a conserved mechanism of host defence}

The term immunothrombosis was originally described by Engelmann and Massberg to refer to an intrinsic effector pathway of innate immunity triggered by pathogens and injured cells to reduce the spread and survival of the invading pathogens ${ }^{10,157}$. By contrast, the term 'thromboinflammation' refers to a process in which inflammation and thrombosis coexist within microvessels in response to harmful stimuli (pathogens, injured cells or other irritants) ${ }^{158}$. Immunothrombosis is mainly triggered by neutrophils and monocytes and is supported by the formation of microthrombi in small vessels, in which endothelial cells exposed to microorganisms adopt a pro-adhesive phenotype ${ }^{159}$.

During immunothrombosis, neutrophils and monocytes release tissue factor and extracellular nucleosomes and degrade endogenous anticoagulants, thereby facilitating activation of coagulation induced by inflammation ${ }^{160}$. Importantly, tissue factor decorates neutrophil extracellular traps (NETs), as found in sepsis ${ }^{161}$ and, more recently, in coronavirus disease 2019 (COVID-19)-related immunothrombosis ${ }^{86}$. NETosis by neutrophils also enhances the activity of the coagulation system by increasing fibrin deposition ${ }^{162,163}$. Histones present in NETs, especially histones $\mathrm{H} 3$ and $\mathrm{H} 4$, can dose-dependently enhance thrombin generation not only by reducing thrombomodulin-mediated protein $\mathrm{C}$ activation ${ }^{164}$ but also by directly activating platelets ${ }^{141}$. Finally, NETs can bind plasma proteins needed for platelet adhesion and thrombus development, such as fibronectin and von Willebrand factor $(\mathrm{VWF})^{141}$. In turn, platelets participate in this process in several ways: they increase the accumulation of immune cells that express tissue factor ${ }^{10,165}$; they bind directly to neutrophils and NETs ${ }^{162,166,167}$; they release damage-associated molecular patterns that promote tissue factor expression within thrombi ${ }^{168}$; and they directly bind to microorganisms for presentation to innate immune cells ${ }^{169,170}$.

Microorganisms have evolved mechanisms to escape immunothrombosis, such as the expression of streptokinase to dissolve fibrin ${ }^{171}$ or nucleases to degrade NETs ${ }^{172,173}$. Some bacteria use immunothrombosis to avoid their killing, such as Staphylococcus aureus, whose staphylocoagulase and VWF-binding protein trigger fibrin generation to form a biofilm and an abscess pseudocapsule ${ }^{174,175}$.

In sum, immunothrombosis can be considered a beneficial mechanism of intravascular immunity. However, when immunothrombosis is uncontrolled, it causes dysregulated activation of the coagulation cascade, leading to microthrombus formation and inflammation, which in turn enhance each other and may, ultimately, develop into thrombosis (thromboinflammation) and disseminated intravascular coagulation.

proposed the occurrence of two or more of the following four criteria in cases of proven COVID-19: a decrease in platelet count $\left(<150 \times 10^{9} \mathrm{l}^{-1}\right)$; an increase in D-dimer levels (more than two times the upper limit of normal); prothrombin time $>1$ s or international normalized ratio $>1.2$; and the presence of thrombosis (macrothrombosis and/or microthrombosis) ${ }^{45}$. The risk of COVID-19-associated coagulopathy is defined by one of the aforementioned four criteria and one of the following ones: increased fibrinogen levels; increased VWF levels (more than two times the upper normal limit); or the presence of lupus anticoagulant and/or high-titre antiphospholipid antibodies ${ }^{45}$. Importantly, COVID-19-associated coagulopathy and risk of COVID-19-associated coagulopathy may further progress and/or predispose to DIC as COVID-19 severity increases.

\section{COVID-19-associated endothelial}

dysfunction. Although COVID-19 should be regarded as a systemic disease, it acutely causes a lung-centred injury primarily affecting the vascular endothelium. COVID19 -associated pneumonia is characterized by infiltration of the lungs with macrophages and neutrophils that cause diffuse lung alveolar damage, the histological equivalent of ARDS ${ }^{46-48}$. Similar findings were previously reported in patients with SARS-CoV ${ }^{49}$. However, the striking finding in COVID-19 compared with SARS-CoV is the more extensive viral infection within the lungs, which results in more diffuse inflammation involving the pulmonary vessels ${ }^{50}$. This extensive infection reflects the particular tropism of SARS-CoV-2 for angiotensin-converting enzyme 2 (ACE2) the cellular receptor for SARS-CoV-2 expressed by type II pneumocytes ${ }^{51}$, which are anatomically close to the lung vascular network and are typically described to be hyperplasic in samples from patients with COVID-19 (REFS ${ }^{52-54}$ ). This hyperplasia of endothelial cells is likely to be caused by lung tissue ischaemia due to congestion of small vessels by inflammatory cells (that is, immunothrombosis) and thrombosis in large vessels ${ }^{55}$.

Endothelial dysfunction was suggested to be an important pathophysiological event in infections by other coronaviruses, which can directly infect endothelial cells ${ }^{56-59}$. SARS-CoV-2 directly infects vascular endothelial cells and leads to cellular damage and apoptosis, thus decreasing the antithrombotic activity of the normal endothelium ${ }^{7,55}$. Alveolar damage, vessel wall oedema, hyaline thrombi, microhaemorrhage 


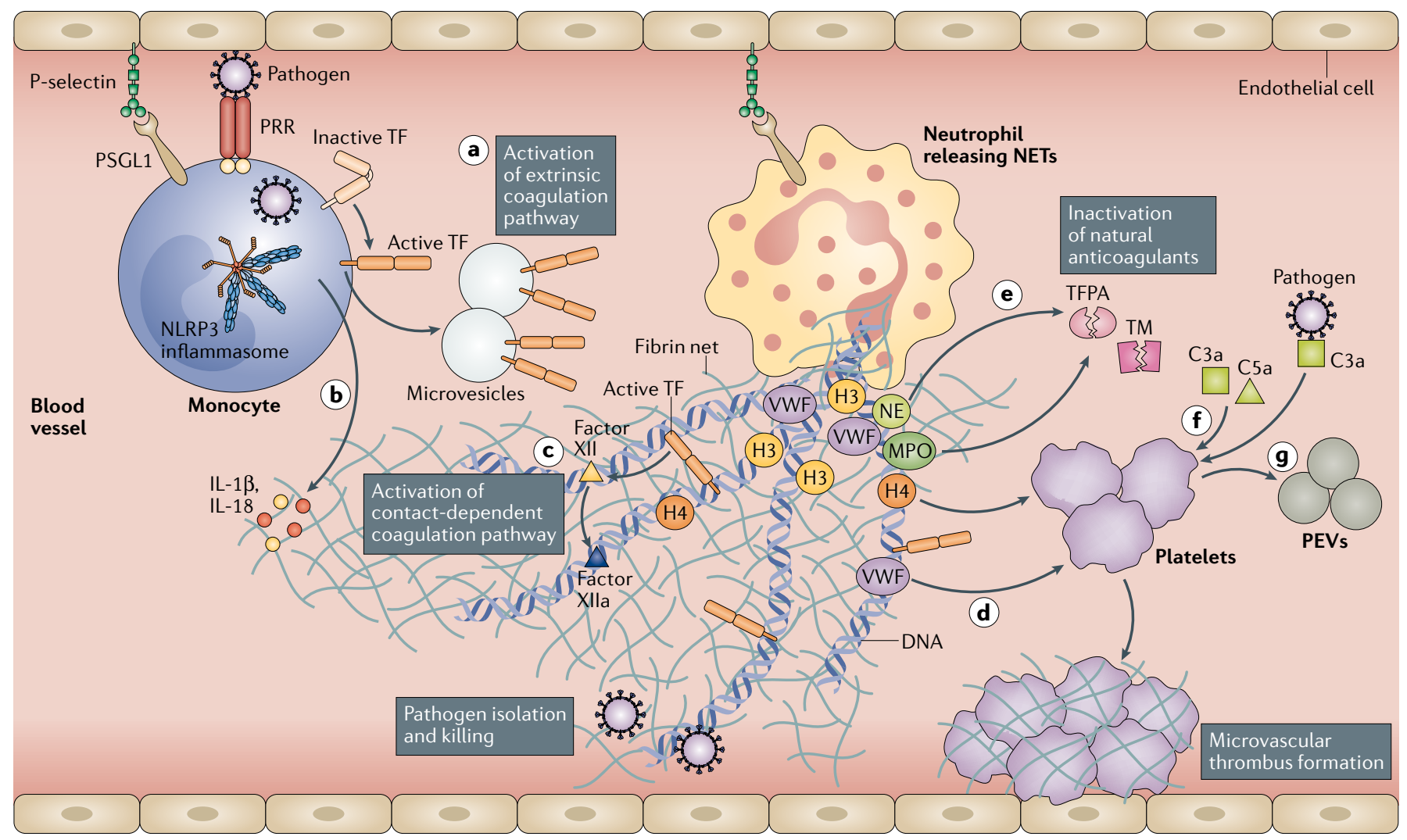

Fig. 1 Immunothrombosis is important in promoting immune defence. Following recognition of a pathogen through pattern recognition receptors (PRRs), monocytes and monocyte-derived microvesicles present activated tissue factor (TF) on their surfaces and release it at sites of pathogen localization, thus activating the extrinsic pathway of coagulation (path a). Pathogens also stimulate the NLRP3 inflammasome in monocytes and/or macrophages, leading to the release of pro-inflammatory cytokines, such as interleukin-1 $\beta$ (IL-1 $\beta$ ) and IL-18 (path b). Neutrophils are recruited and contribute to this process through the release of neutrophil extracellular traps (NETs), which directly activate factor XII and, thus, the contactdependent pathway of coagulation (path c). NETs also bind von Willebrand factor (VWF) and help to recruit platelets (path d). Histones, in particular $\mathrm{H} 3$ and $\mathrm{H} 4$, trigger activation of platelets. In addition, neutrophil elastase (NE) and myeloperoxidase (MPO) in NETs cleave and inactivate natural anticoagulants (tissue factor pathway inhibitor (TFPA) and thrombomodulin (TM)) (path e). Finally, NETs can externalize and bind TF, promoting activation of the extrinsic pathway of coagulation. Platelets support the immunothrombotic process by activating the contact-dependent pathway of coagulation through the release of polyphosphates and, along with endothelial cells, may promote fibrin generation. Platelets can also be activated by $\mathrm{C} 3 \mathrm{a}$ and $\mathrm{C} 5 \mathrm{a}$ (path $\mathbf{f}$ ). Activated platelets release large amounts of pro-inflammatory cytokines in platelet extracellular vesicles (PEVs) (path g). Through this mechanism, pathogens such as severe acute respiratory syndrome coronavirus 2 (SARS-CoV-2) become trapped within the fibrin-based NETs and killed. The immunothrombotic process allows pathogen killing to be restricted to the intravascular compartment, thus limiting injury to organs. Although it is clear that immunothrombosis participates in SARS-CoV-2 pathogenesis, the exact mechanisms are still under investigation. These may include the following: direct injury of endothelial cells by the virus and consequent activation of the coagulation cascade; infiltration of neutrophils that lead to NET formation; induction of hypoxaemia causing upregulation of TF expression by hypoxia-inducible transcription factors and formation of clots; activation of complement that promotes coagulation and recruits and activates platelets, monocytes and neutrophils, thus triggering TF expression; and an abnormal increase in the levels of pro-inflammatory cytokines causing direct cell damage. and diffuse thrombosis of peripheral small vessels have emerged as key features of COVID-19 contributing to respiratory failure $^{6,8,46}$ (FIG. 2).

Endothelial cells of lung blood vessels can be activated by the high levels of pro-inflammatory cytokines (IL-1, IL-6 and TNF) and ferritin in severe COVID-19 $\left(\right.$ REF. $\left.^{60}\right)$. Moreover, increased levels of the endothelial adhesion protein VWF were reported both in patients admitted to the ICU and in non-critically ill patients ${ }^{34,35,61,62}$, and this increase seems to be associated with a mild reduction in the activity of a disintegrin and metalloproteinase with a thrombospondin type 1 motif, member 13
(ADAMTS13), a metalloproteinase that regulates the size of VWF multimers ${ }^{63}$. Endothelial cell perturbation along with the release of large VWF multimers and relative insufficient VWF cleavage owing to ADAMTS13 consumption may account for increased platelet-vessel wall interactions causing thrombotic microangiopathy. Indeed, a relative deficiency in ADAMTS13 activity was previously described in inflammatory conditions with high levels of IL-6, as found in patients with SARS-CoV-2 infection ${ }^{64}$. Other markers of endothelial cell injury have been recently investigated. Higher levels of soluble P-selectin (a marker of endothelial and platelet activation) were observed in patients admitted to the ICU than in patients not in the ICU, whereas increased levels of thrombomodulin (a specific marker of endothelial activation generally released during endothelial cell injury) were associated with increased mortality risk $^{35}$. Higher numbers of circulating endothelial cells were described in patients with COVID-19, especially among those admitted to the ICU, and the presence of circulating endothelial cells positively correlated with platelet and lymphocyte counts and with the classical endothelial marker soluble vascular cell adhesion molecule 1 (sVCAM1) ${ }^{65}$. This was recently confirmed by another report from Italy, 
showing that patients with COVID-19 had increased circulating endothelial cells and higher plasma levels of soluble intercellular adhesion molecule 1 (sICAM1) and sVCAM1, further supporting the concept of endothelial dysfunction in COVID-19 $\left(\right.$ REF. $\left.^{66}\right)$.

Considering the important role of the endothelium in regulating haemostasis, fibrinolysis and vessel wall permeability,

\section{A}
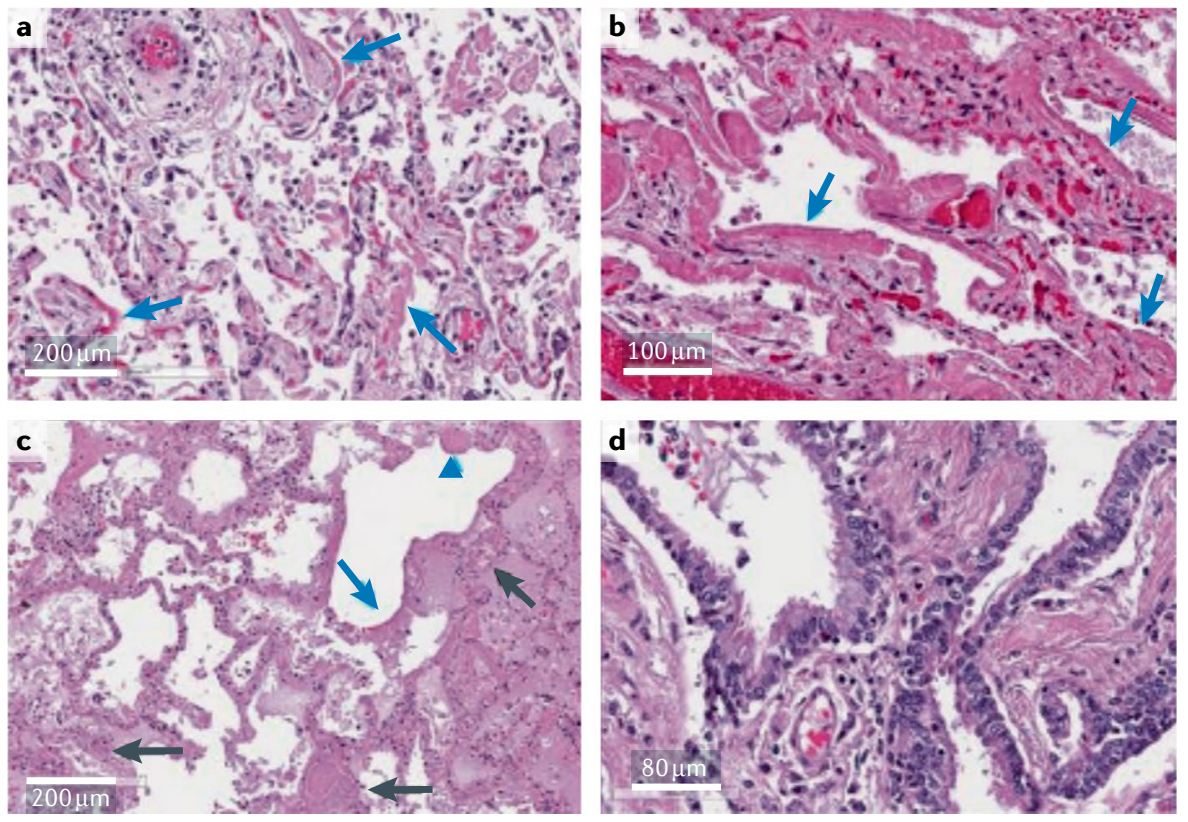

B
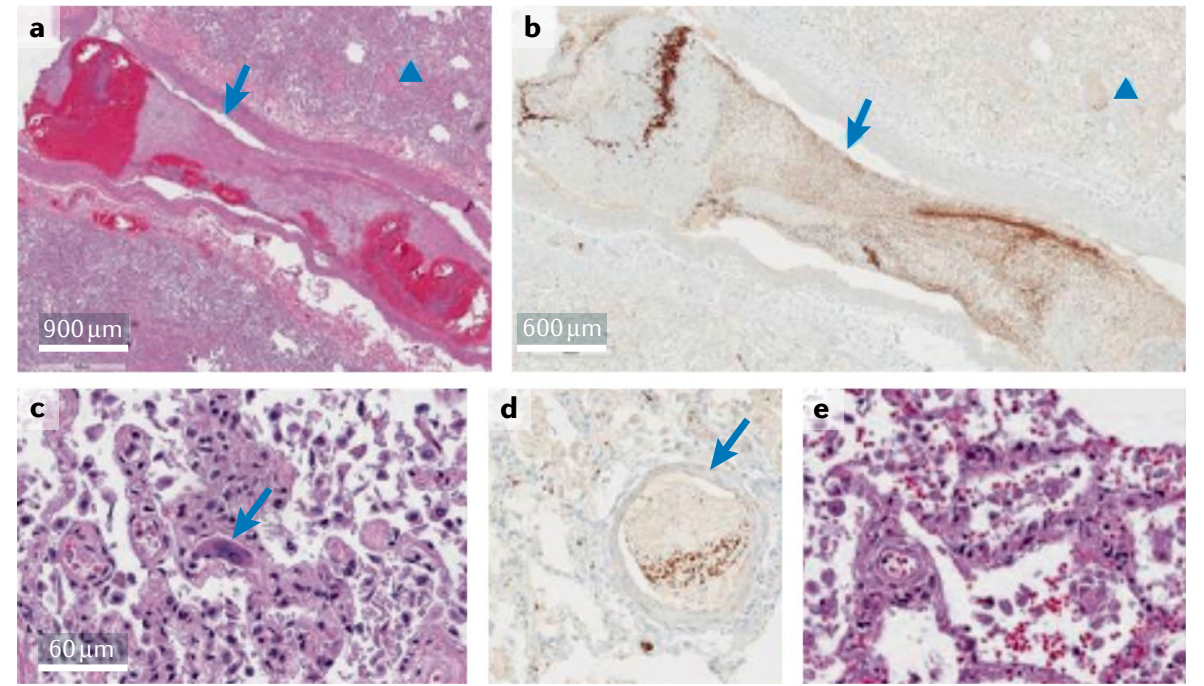

Fig. 2 | Seminal pathology features of patients with COVID-19. A | Hyaline membrane formation is a typical finding in patients with alveolar damage irrespective of being ventilated or not (blue arrows in panels $\mathbf{a}$ and $\mathbf{b}$ ). Organizing fibrosis (blue arrowhead) and fibrin thrombi in small blood vessels (black arrows) with oedema, along with extensive haemorrhage (haematoxylin and eosin staining) and hyaline membranes (blue arrows) (panel c). The bronchial respiratory mucosa is almost entirely intact and no squamous metaplasia is evident (panel d), different to that observed in patients with severe acute respiratory syndrome coronavirus 2 (SARS-CoV-2). B | Two thrombi, one in a small pulmonary artery (blue arrow) and one in a pulmonary venule (blue arrowhead), in the haematoxylin and eosin staining (panel a) and in the CD61 immunostaining for platelets within thrombi (panel b). Megakaryocytes within small vessels and alveolar capillaries (panel c, blue arrow). CD61 immunostaining of a fibrin- and platelet-rich thrombus in a small vessel (panel d), with a megakaryocyte stained below (blue arrow). Small, perivascular aggregates of lymphocytes (panel e). COVID-19, coronavirus disease 2019. Images reprinted from The Lancet, 8, Fox, S. E. et al., Pulmonary and cardiac pathology in African American patients with COVID-19: an autopsy series from New Orleans, 681-686, Copyright (2020), with permission from Elsevier (REF. ${ }^{6}$.

endothelial dysfunction in pulmonary microvessels acts as a trigger for immunothrombosis, resulting in the coagulopathy observed in patients with COVID-19 (REFS ${ }^{27,47,67,68}$ ). In addition, endothelial dysfunction is recognized as a risk factor of microvascular dysfunction through a shift towards vasoconstriction, by promoting ischaemia, inflammation and a procoagulant state $^{69}$. In addition, the inflammatory environment triggers the expression of activated tissue factor on endothelial cells, macrophages and neutrophils, which amplifies activation of the coagulation cascade within the lungs ${ }^{60}$. These events fuel a positive immunothrombotic feedback loop in which hyperinflammation triggers the hypercoagulant state, with thrombosis occurring within pulmonary microvessels. Lastly, platelet activation within the microvasculature is increased in patients with severe COVID-19 and is associated with poor outcomes ${ }^{70,71}$. Plateletvessel wall interactions, mediated by surface receptors (integrins and selectins) and adhesive proteins (VWF and fibrinogen), impair vessel wall integrity or increase endothelial injury, ultimately leading to microvascular obstruction ${ }^{72}$. Moreover, platelets were found to be hyperactivated in patients with COVID-19 and to be a source of procoagulant extracellular vesicles $^{73}$ and polyphosphates, which can exacerbate the thromboinflammatory cascade via neutrophils ${ }^{74}$. Of importance, activated platelets are critically involved in neutrophil extracellular trap (NET) formation $^{71}$, which is an essential element of immunothrombosis. All of these elements support the hypothesis that endothelial dysfunction, or endotheliopathy ${ }^{35,75}$, and platelet activation are key features of COVID-19-associated coagulopathy and may mediate the damage leading to severe disease.

COVID-19-associated ARDS. COVID19-related ARDS is considered atypical, with its main characteristic being a dissociation between hypoxaemia severity and relatively good respiratory mechanics ${ }^{76,77}$. From a clinical standpoint, two phenotypes are described ${ }^{67,78}$. Patients with 'type L' have high lung compliance, lower lung weight as estimated by computed tomography scan and poor response to positive end-expiratory pressure. Patients with 'type $\mathrm{H}$ ' are those with a deteriorating disease or suboptimally treated disease that present with areas of accumulation of inflammatory exudate in the alveoli as 


\section{Box 2 | The role of complement in sepsis}

The complement system is an important component of the innate immune system and activates following infections. After complement activation (through the classic, alternative or lectin pathways), the common pathway is responsible for the production of $\mathrm{C} 3 \mathrm{a}$ and $\mathrm{C} 5 \mathrm{a}$, then stimulating formation of the C $5 b-9$ membrane attack complex, which causes the lysis of pathogen cells ${ }^{176}$. In a mouse model of intranasal infection with severe acute respiratory syndrome coronavirus (SARS-CoV), early activation of the complement system, especially complement component C3, caused acute respiratory distress syndrome (ARDS) ${ }^{177}$. However, C3-deficient mice experienced reduced respiratory dysfunction, limited neutrophil and monocyte lung infiltration, and showed lower levels of cytokines both in the lungs and the blood ${ }^{177}$. Excessive activation of complement in the advanced phases of sepsis may therefore cause tissue injury contributing to organ failure.

The complement and coagulation pathways are closely linked ${ }^{178,179}$. In particular, complement end products can trigger the release of procoagulant products and reduce the production of natural anticoagulants ${ }^{178}$. Additionally, the binding of $\mathrm{C} 1 \mathrm{q}$ to its receptor on platelets triggers the expression of integrins and $\mathrm{P}$-selecti ${ }^{180}$, whereas $\mathrm{C} 3$ stimulates platelet activation and aggregation. The activation of $\mathrm{C} 5$ during the inflammatory response is responsible for the expression of tissue factor on leukocytes and endothelial cells ${ }^{18,182}$. Complement may indirectly induce procoagulant changes; for example, activated $\mathrm{C} 3$ and $\mathrm{C} 5$ contribute to cytokine release soon after the innate immune response starts, particularly increasing the concentrations of tumour necrosis factor (TNF) and interleukin-6 (IL-6).

observed through computed tomography scan, low lung compliance, greater lung weight and good response to positive end-expiratory pressure. This clinical picture couples with endothelial damage, breaking down pulmonary vasoregulation, stimulating ventilation-perfusion mismatch - which is mainly responsible for hypoxaemia - and supporting immunothrombosis. In this respect, the events leading to atypical ARDS can be summarized by the term 'microvascular COVID-19 lung vessels obstructive thromboinflammatory syndrome' (MicroCLOTS) ${ }^{79}$. According to this proposed definition, ARDS is the result of a hyperimmune reaction of the host to SARS-CoV-2 that is both directly mediated by the virus through cellular injury and release of pro-inflammatory signals and indirectly mediated by complement activation (BOX 2), leading to leukocyte recruitment and increased release of pro-inflammatory cytokines. This results in a severe tissue injury with vascular endothelial dysfunction and alveolar cell damage, which may explain the progressive worsening of ventilationperfusion mismatch and pulmonary microthrombosis ${ }^{79}$.

\section{NETs in COVID-19-associated}

immunothrombosis. NETs are likely to be important players in deregulated immunothrombosis in COVID-19 (REF ${ }^{80}$ ). Indeed, ARDS can be considered as a NETopathy ${ }^{81}$; higher levels of NETs were described in the plasma and bronchoalveolar fluid of patients with transfusion-associated and pneumonia-related ARDS compared with those without ARDS $^{82-84}$. NETs are markers of disease severity in patients with
SARS-CoV-2 infection ${ }^{80,85}$. Compared with controls, patients with COVID-19 have higher levels of serum ${ }^{85}$ or plasma ${ }^{80}$ myeloperoxidase (MPO)-DNA complexes, which are biomarkers of circulating NET fragments, and further increases were found in those patients requiring invasive mechanical ventilation. MPO-DNA complexes and thrombinantithrombin complexes were positively correlated in patients with COVID-19, and the anticoagulant dabigatran or complement targeting inhibited NETosis ${ }^{86}$, further suggesting a central role for NETs in the immunothrombotic milieu. MPO-DNA levels return to normal in convalescent plasma, indicating that this is a transient increase $^{80}$. High-dimensional flow cytometric analysis of circulating neutrophils revealed a highly activated phenotype in patients with most severe COVID-19 (REF. ${ }^{71}$ ). Neutrophils can be found embedded in fibrin clots and in close association with platelets within microthrombi in the lungs $\mathrm{s}^{80}$, heart and kidneys of patients with COVID-19 $\left(\mathrm{REF}^{71}\right)$. In severe disease, neutrophils adopted the so-called low-density phenotype that is more prone to spontaneously form NETs; this greater NETosis was linked to increased intravascular aggregation, leading to microthrombosis and organ damage ${ }^{87}$. NETs have also been identified in the lung parenchyma and alveolar space in autopsy case reports ${ }^{9,88}$. Interestingly, neutrophils from healthy donors showed increased adhesion to activated platelets in plasma from patients with COVID-19 compared with that from control patients, causing enhanced NET generation $^{71}$. Indeed, both serum and plasma from patients with COVID-19 strongly stimulate the production of NETs from healthy neutrophils ${ }^{71,80,85}$. In addition, neutrophils isolated from patients with COVID-19 showed elevated NET release at baseline similar to phorbol myristate acetate (PMA)-stimulated neutrophils from healthy donors, indicating that the COVID-19 plasma environment promotes NET formation ${ }^{80}$. The virus itself is also reported to directly activate neutrophils towards NETosis, promoting epithelial cell death ${ }^{89}$. NETs may provide a scaffold for the deposition of $\mathrm{C} 3$, properdin and factor $\mathrm{B}$, thus directly participating in complement activation $^{90}$. This mechanism may fuel an amplifying feedback loop promoting further NET formation and platelet and endothelial cell activation ${ }^{91}$. The observation of antiphospholipid antibodies, which can directly stimulate NETosis ${ }^{92}$, in patients with COVID-19, as well as in other infections ${ }^{93}$, suggests that SARS-CoV-2 infection might synergize with antiphospholipid antibodies to promote the immunothrombotic process. Indeed, higher titres of antiphospholipid antibodies were associated with increased activity of neutrophils and platelets and more severe respiratory disease ${ }^{94}$.

Whether NETs are direct contributors to the dysregulated cytokine response is still unclear. Indeed, NETs participate in acute lung injury by inducing macrophage release of IL- $1 \beta$, which in turn can increase NET formation ${ }^{95,96}$. In addition, most inflammatory mediators that are increased in patients with COVID-19 are known to regulate neutrophil activity through the expression of chemotactic products $^{16,97}$. These data suggest that dysregulated cytokine release might be sustained by crosstalk between neutrophils and macrophages mediated by NETs, resulting in a deranged or exaggerated immunothrombotic status.

\section{COVID-19: a syndrome of deregulated} immunothrombosis? On the basis of the aforementioned evidence, we propose that exaggerated immunothrombosis, occurring for the most part within lung microvessels, drives the clinical manifestations of COVID-19 (REF. ${ }^{3}$ ), although systemic viraemia early in the disease course may also be important ${ }^{98}$ (FIG. 1). The occurrence of severe events, such as acute respiratory failure and ARDS, may be explained by a deregulated process of immunothrombosis involving the pulmonary microcirculation. In support of this, there is increasing evidence that COVID-19 is a disease of the endothelium ${ }^{35}$ that results in elevated levels of PAI1 and VWF, increased platelet 
activation and a state of hypercoagulability, with consequent venous, arterial and microvascular thrombosis. The exact factors triggering this endotheliopathy have not yet been recognized, but potentially include the virus itself (causing endothelial cell injury that triggers the coagulation cascade involving fibrin and platelets ${ }^{47,99}$ ), immune cell (neutrophil and/or macrophage and platelet $^{73,100,101}$ ) infiltration, hypoxaemia (that can induce hypoxia-inducible transcription factors that upregulate tissue factor expression ${ }^{102}$ and lead to fibrin-based clot formation, thus supporting a thromboinflammatory feedback loop), complement-mediated damage or a surge in pro-inflammatory cytokine (such as IL- $1 \beta$ and IL-6) release with direct cell damage.

\section{Thrombosis management in COVID-19}

If we consider COVID-19 as a vascular disease primarily involving the endothelium, an ideal therapeutic approach would be both antithrombotic and anti-inflammatory. Here, we describe some of the main approaches that are being explored to address these features of COVID-19 (TABLE 1).

\section{Antithrombotic therapies. As the} coagulation cascade is dysregulated in COVID-19, anticoagulation approaches have been explored for the treatment, particularly of patients with incident venous thromboembolism (VTE) or acute coronary syndrome due to plaque rupture $^{103}$. Indeed, a study reported that $40 \%$ of patients hospitalized with COVID-19 are at high risk of $\mathrm{VTE}^{104}$ and data from a large retrospective cohort of patients in the United States suggested that anticoagulation may reduce mortality, especially among those with severe disease requiring invasive mechanical ventilation ${ }^{105}$. Pharmacological VTE prophylaxis, including daily low molecular weight heparin or twice-daily subcutaneous unfractionated heparin, may also be of benefit to patients hospitalized with COVID-19 who are bedridden or need intensive care, or patients discharged from hospital but with relevant risk of VTE that is, individuals with reduced mobility, pro-thrombotic comorbidities (active cancer or obesity), older age, a history of VTE and persistently elevated levels of D-dimer ${ }^{103,106,107 .}$.

Studies of heparin treatment of COVID-19 have shown positive results ${ }^{103,106}$ (Supplementary Table 1), and this is now one of a growing number of agents available to manage COVID-19 either as a prophylactic or therapeutic regimen ${ }^{108}$ (TABLE 1). The use of fondaparinux (a selective indirect antithrombin-dependent factor Xa inhibitor) in patients with COVID-19 was reported in two papers, with contrasting results about its safety ${ }^{109,110}$. The same applies to direct oral anticoagulants, the use of which in patients with COVID-19 has been little studied to date ${ }^{111,112}$. Several trials of heparin and other anticoagulant agents are in progress: the Randomized, Embedded,
Multi-factorial Adaptive Platform Trial for Community-Acquired Pneumonia (REMAP-CAP) (NCT02735707); the Accelerating COVID-19 Therapeutic Interventions and Vaccines-4 (ACTIV-4) trial (NCT04505774); and the Antithrombotic Therapy to Ameliorate Complications of COVID-19 (ATTACC) trial (NCT04372589). These trials are

\section{Glossary \\ Antithrombin \\ A glycoprotein produced by the liver that inactivates different enzymes of the coagulation cascade. Antithrombin activity is strongly increased by the anticoagulant medication heparin, which enhances \\ Microthrombosis \\ The formation of thrombi (clots) within small vessels, which is typical of the immunothrombotic process. Microthrombosis is often encountered in patients with coronavirus disease 2019 (COVID-19).} the binding of antithrombin to factor Ila (thrombin) and factor $\mathrm{Xa}$

\section{Cytokine storm}

A sudden surge in circulating levels of pro-inflammatory cytokines, such as interleukin-1 (IL-1), IL-6, tumour necrosis factor (TNF) and interferon- $\gamma$ (IFN $\gamma$ ) Clinically, this can result in hypotension, acute renal failure, impaired pulmonary function and even death

\section{D-dimer}

A small protein derived from fibrin degradation that is found in the blood once a blood clot is degraded. The name comes from the presence of two $D$ fragments of the fibrin protein united by a cross-link

Disseminated intravascular coagulation (DIC). An acquired syndrome characterized by the intravascular activation of coagulation without a precise localization in response to microvascular injury, leading to organ dysfunction when sufficiently severe. DIC involves large production of thrombin accompanied by platelet activation and consumption, altered fibrinolysis and microvascular thrombosis. It can occur in critically ill patients with overwhelming infection, fulminant sepsis, extensive tissue damage or malignancy.

\section{Haemostasis}

The combination of events that result in cessation of bleeding. Haemostatic processes include constriction of blood vessels, platelet aggregation and coagulation (clotting) of the blood

\section{Hyaline thrombi}

Clots occurring in small vessels due to the formation of hyaline membranes and surfactant dysfunction after an inflammatory injury. These formations are the pathological equivalent of acute respiratory distress syndrome (ARDS) occurring in patients with severe-to-critical coronavirus disease 2019 (COVID-19).

\section{Lung compliance}

A measure of the ability of the lungs to stretch and expand. A low compliance refers to that of a stiff lung, as occurs in fibrosis. A high compliance means a flexible lung, as seen in emphysema.

Macrophage activation syndrome A syndrome in which a dysregulated activation and proliferation of macrophages and T cells leads to increased release of pro-inflammatory cytokines.

\section{NLRP3 inflammasome}

Macromolecular structure that is responsible for sensing danger and triggering a local or systemic inflammatory response. Following NLRP3 inflammasome activation, large quantities of pro-inflammatory cytokines (in particular IL-1 $\beta$ ) are released for extracellular secretion. These cytokines are important in the inflammatory response and responsible for symptoms such as fever.

Neutrophil extracellular trap

(NET). Fibrillary structure consisting of DNA complexed with histones ( $\mathrm{H} 3$ and $\mathrm{H} 4)$ and proteins from neutrophil granules, especially neutrophil elastase (NE), myeloperoxidase (MPO) and cathepsin G. NETs act as a mesh that traps microorganisms and exposes them to neutrophil-derived thrombus formation and activation of coagulation.

Positive end-expiratory pressure Pressure within the lung alveoli above atmospheric pressure that is recorded at the end of expiration. Besides intrinsic positive end-expiratory pressure (caused by an incomplete exhalation), extrinsic positive end-expiratory pressure is provided by a ventilator during mechanical ventilation and is meant to reduce end-expiratory alveolar collapse, thus improving hypoxaemia or reducing ventilator-associated lung injury in patients with acute respiratory distress syndrome (ARDS).

Thrombosis

The formation of a thrombus (clot) inside blood vessels, resulting in partial or complete vessel occlusion.

Thrombotic microangiopathy

Pathology characterized by formation of thrombi in capillaries owing to endothelial damage. In particular, decreased levels of the protease a disintegrin and metalloproteinase with a thrombospondin type 1 motif, member 13 (ADAMTS13) lead to an accumulation of ultra-large von Willebrand factor (VWF) multimers, which promote platelet-vessel wall interactions, causing thrombus formation. The clinical presentation comprises multiple organ dysfunctions, especially in the brain and kidneys.

Ventilation-perfusion mismatch Impairment of the ventilation to perfusion ratio, meaning that a part of the lung is adequately oxygenated but not perfused, or vice versa, caused by diseases such as pneumonia and pulmonary embolism.

Von Willebrand factor

(VWF). The main adhesive protein mediating interactions between platelets and the vessel wall, leading to platelet aggregation. Preformed multimers are released from Weibel-Palade bodies of activated endothelial cells. effector molecules. NETs also provide a scaffold for 
Table 1 | Putative therapeutic approaches for COVID-19

\begin{tabular}{|c|c|c|c|c|}
\hline Therapeutic agent & Target & Outcome & Clinical trial & Refs \\
\hline \multicolumn{5}{|l|}{ Antithrombotic drugs } \\
\hline Heparin & $\begin{array}{l}\text { Inactivation of thrombin and } \\
\text { activated factor } X \text { through an } \\
\text { antithrombin-dependent mechanism }\end{array}$ & $\begin{array}{l}\text { Controversial evidence on mortality } \\
\text { and need for mechanical ventilation } \\
\text { from observational studies }\end{array}$ & - & $105,152-154$ \\
\hline Garadacimab & Factor II blockade & One study is ongoing & NCT04409509 & - \\
\hline $\begin{array}{l}\text { Nafamostat } \\
\text { mesylate }\end{array}$ & $\begin{array}{l}\text { Serine protease inhibitor of thrombin, } \\
\text { plasmin and trypsin }\end{array}$ & $\begin{array}{l}\text { Ongoing trials based on in vitro } \\
\text { blockade of MERS-CoV infection }\end{array}$ & NCT04352400, NCT04418128 & - \\
\hline $\begin{array}{l}\text { Tissue-type } \\
\text { plasminogen } \\
\text { activator }\end{array}$ & $\begin{array}{l}\text { Conversion of plasminogen into } \\
\text { plasmin, leading to breakdown of } \\
\text { cross-linked fibrin }\end{array}$ & $\begin{array}{l}\text { A phase lla trial in patients with } \\
\text { COVID-19-related ARDS is ongoing }\end{array}$ & NCT04357730 & 119 \\
\hline \multirow[t]{2}{*}{ Dipyridamole } & $\begin{array}{l}\text { Phosphodiesterase inhibitor } \\
\text { reversibly inhibiting platelet } \\
\text { aggregation; in addition, it potentiates } \\
\text { vascular-protective effects of } \\
\text { endothelium-derived nitric oxide }\end{array}$ & $\begin{array}{l}\text { In vitro suppression of SARS-CoV-2 } \\
\text { replication and improvement of } \\
\text { lung pathology in a model of viral } \\
\text { pneumonia }\end{array}$ & \multirow[t]{2}{*}{ NCT04391179 } & \multirow[t]{2}{*}{$121,124,125$} \\
\hline & $\begin{array}{l}\text { Possible activity against } \\
\text { positive-stranded RNA viruses and } \\
\text { prevention of NET formation }\end{array}$ & A trial is ongoing & & \\
\hline Ticagrelor & $\begin{array}{l}\mathrm{P} 2 \mathrm{Y}_{12} \text {-receptor antagonist and } \\
\text { attenuation of NET formation }\end{array}$ & Ongoing trials & NCT02735707, NCT04518735 & - \\
\hline \multicolumn{5}{|c|}{ Anti-inflammatory drugs } \\
\hline \multirow[t]{2}{*}{ NE inhibitors } & \multirow[t]{2}{*}{$\begin{array}{l}\text { NE inhibition blocks NET formation } \\
\text { and reduces SARS-CoV- } 2 \text { spike protein } \\
\text { proteolytic activation }\end{array}$} & $\begin{array}{l}\text { Sivelestat has been approved to treat } \\
\text { ARDS in Japan and South Korea, but } \\
\text { findings are inconsistent }\end{array}$ & \multirow[t]{2}{*}{-} & \multirow[t]{2}{*}{$131-133$} \\
\hline & & $\begin{array}{l}\text { No current indication is available for } \\
\text { SARS-CoV-2-related ARDS }\end{array}$ & & \\
\hline DNase I & Dissolution of NETs & $\begin{array}{l}\text { Dornase alpha has been tested in } \\
\text { two case studies in patients with } \\
\text { COVID-19-associated ARDS }\end{array}$ & $\begin{array}{l}\text { NCT04402944, NCT04355364, } \\
\text { NCT04432987, NCT04359654, } \\
\text { NCT04445285, NCT04402970 }\end{array}$ & 138,139 \\
\hline Ruxolitinib & JAK1/2 inhibitor & A trial is ongoing in Germany & NCT04338958 & $142,155,156$ \\
\hline \multirow[t]{3}{*}{$\begin{array}{l}\text { Eculizumab and } \\
\text { ravulizumab }\end{array}$} & \multirow[t]{3}{*}{ C5 inhibitors blocking MAC formation } & $\begin{array}{l}\text { Eculizumab showed good efficacy in } \\
\text { reducing inflammatory biomarkers }\end{array}$ & \multirow[t]{3}{*}{$\begin{array}{l}\text { NCT04288713, NCT04369469, } \\
\text { NCT04390464 }\end{array}$} & \multirow[t]{3}{*}{147,148} \\
\hline & & $\begin{array}{l}\text { In ICU patients, eculizumab improved } \\
\text { 15-day survival and hypoxia } \\
\text { compared with SOC }\end{array}$ & & \\
\hline & & $\begin{array}{l}\text { Trials with eculizumab and } \\
\text { ravulizumab are ongoing }\end{array}$ & & \\
\hline \multirow[t]{2}{*}{ AMY-101 } & \multirow[t]{2}{*}{$\begin{array}{l}\text { Inhibition of } \mathrm{C} 3 \text { cleavage by direct } \\
\text { binding }\end{array}$} & $\begin{array}{l}\text { Limited experience is now available, } \\
\text { with encouraging results }\end{array}$ & \multirow{2}{*}{$\begin{array}{l}\text { EudraCT number 2020-001550- } \\
\text { 22, EudraCT number 2020- } \\
\text { 004408-32, NCT04395456 }\end{array}$} & \multirow[t]{2}{*}{149} \\
\hline & & $\begin{array}{l}\text { Three phase II clinical trials in patients } \\
\text { with COVID-19-associated ARDS are } \\
\text { ongoing }\end{array}$ & & \\
\hline
\end{tabular}

ARDS, acute respiratory distress syndrome; COVID-19, coronavirus disease 2019; ICU, intensive care unit, JAK, Janus kinase; MAC, membrane attack complex; MERS-CoV, Middle East respiratory syndrome coronavirus; NE, neutrophil elastase; NET, neutrophil extracellular trap; RCT, randomized controlled trial; SARS-CoV-2, severe acute respiratory syndrome coronavirus 2; SOC, standard of care.

testing whether the use of full-dose versus low-dose unfractionated heparin or low molecular weight heparin benefits patients with COVID-19 requiring ICU admission.
However, enrolment of critically ill patients has been temporarily stopped owing to futility and safety concerns for this subgroup of patients. Response to this anticoagulant therapy may depend on the time of administration; if given too late, thrombi in the microcirculation and large vessels may already be too extensive. 
An alternative approach to pharmacologically disrupt the coagulation cascade involves blockade of factor XII (FXII), which is responsible for activation of the contact pathway (TABLE 1). FXII blockade has been shown to protect from occlusive thrombosis in animal models without impairing haemostasis ${ }^{113,114}$. Thus, a FXII-blocking antibody (garadacimab) is currently being explored in a multicentre, double-blind, placebo-controlled phase II trial in patients with respiratory distress and COVID-19 (NCT04409509). Similarly, a serine protease inhibitor of thrombin, plasmin and trypsin known as nafamostat mesylate (used in Japan for the treatment of pancreatitis and DIC and the prevention of clots during dialysis ${ }^{115}$ ) is being investigated in clinical trials for COVID-19 (NCT04352400, NCT04418128). This drug showed potential to block Middle East respiratory syndrome coronavirus infection in vitro ${ }^{116}$ (TABLE 1).

The hypofibrinolytic state observed in ARDS has been targeted by the use of tissue-type plasminogen activator, which is responsible for the conversion of plasminogen into plasmin, leading to the breakdown of cross-linked fibrin ${ }^{117,118}$. Indeed, tissue-type plasminogen activator infusion showed benefit in three patients with severe COVID-19 (REF. ${ }^{119}$ ), and a phase IIa trial in patients with COVID-19-related ARDS is currently ongoing (NCT04357730).

The antiplatelet agent dipyridamole may have therapeutic potential ${ }^{120}$ (TABLE 1). This agent, apart from its antiplatelet function, was shown to provide broad-spectrum antiviral activity (especially against positive-stranded RNA viruses), suppress inflammation and favour mucosal healing, and prevent acute injury and fibrosis in the lungs, heart and kidney ${ }^{121-123}$. Dipyridamole treatment in patients with COVID-19 may also have the added benefit of preventing NETosis by promoting $3^{\prime}, 5^{\prime}$-cyclic adenosine monophosphate (cAMP) generation in neutrophils, as was shown in the context of antiphospholipid syndrome ${ }^{124}$. Liu et al. demonstrated that dipyridamole can suppress SARS-CoV-2 replication in vitro and improve lung pathology in an animal model of viral pneumonia through a type I interferon response ${ }^{125}$. This drug was tested in 14 patients and led to an improvement in clinical status compared with controls ${ }^{125}$. A trial of dipyridamole is currently ongoing (NCT04391179). Another antiplatelet agent, ticagrelor, showed the ability to attenuate NET formation ${ }^{126}$. Accordingly, two trials of ticagrelor are ongoing or recently completed (NCT02735707, NCT04518735).
Anti-inflammatory therapies.

Immunomodulatory therapies, such as inhibitors of IL-6, IL-1, GM-CSF and Janus kinases (JAKs), aim to blunt the cytokine dysregulation associated with severe COVID-19 (REFS ${ }^{15,127,128}$ ) (TABLE 1), but little is known about their impact on the thrombotic complications of the disease.

Given that NETs have a direct role in the immunothrombotic process in COVID-19, blocking NETs may help to improve prognosis (TABLE 1). Some drugs targeting NETs already exist or are under development, such as inhibitors of neutrophil elastase (NE), PAD4 and gasdermin $\mathrm{D}^{129}$. For example, $\mathrm{NE}$ inhibitors can block NET formation, limit the toxic activities of NE itself on lung connective tissue and reduce spike protein proteolytic activation that is responsible for SARS-CoV-2 binding to host cells ${ }^{130}$. Sivelestat, an NE inhibitor, has been approved to treat ARDS in Japan and South Korea, but findings are inconsistent as the severity of lung injury is likely to represent the most important predictor for patient outcome $^{131-133}$. However, no evidence is currently available in favour of using NE inhibitors for COVID-19-induced ARDS.

NETs can be degraded by recombinant DNase I (dornase alpha), which is currently given by inhalation to patients with cystic fibrosis to target NETs in the airways ${ }^{134}$. Dornase alpha may help to dissolve respiratory secretions in patients with COVID-19 (REF. ${ }^{135}$ ), reducing the risk for secondary infections and improving ventilation, and may also reduce the risk of progression towards ARDS, as shown in animal models ${ }^{136,137}$. Two case studies have shown that administration of dornase alpha is well tolerated in patients with COVID-19associated ARDS ${ }^{138,139}$, and several trials are underway to determine the efficacy of this approach (NCT04402944, NCT04355364, NCT04432987, NCT04359654, NCT04445285, NCT04402970). Nebulizer administration of DNase provides direct access to NETs within the alveolar space, but there may be a barrier to reaching systemic efficacy owing to the high reported levels of platelet factor 4 (PF4) in severe COVID-19 $\left(\right.$ REF $\left.^{80}\right)$. PF4 binding to NETs leads to their compaction and decreased susceptibility to DNase degradation ${ }^{140}$. Therefore, DNase administration is unlikely to ameliorate NET-mediated thrombosis, although the combination with heparin (which also digests $\mathrm{NETs}^{141}$ ) may be able to overcome this DNase resistance.

Other promising approaches to prevent NET formation rather than target already released NETs include JAK1/2 inhibition with ruxolitinib ${ }^{142}$, dipyridamole ${ }^{124}$, ticagrelor ${ }^{126}$ and neonatal NET-inhibitory factor, an endogenous peptide produced in newborns that restricts the ability of neutrophils to produce NETs ${ }^{143}$. Neonatal NET-inhibitory factor was shown to prevent COVID-19 plasma-induced NET formation by healthy donor neutrophils in vitro, but the mechanism of action remains to be elucidated $^{80}$.

Finally, evidence is accumulating on the role of complement activation in the pathogenesis and severity of SARS-CoV-2 (REF. ${ }^{144}$ ) as well as on complement-mediated thrombotic microangiopathy in patients with COVID-19 (REF. ${ }^{145}$ ). Thus, therapies for thrombotic microangiopathy complement inhibitors - may be used in this setting. C3 blockade can inhibit the production of both $\mathrm{C} 3 \mathrm{a}$ and $\mathrm{C} 5 \mathrm{a}$, but also of intrapulmonary C3a and the release of IL-6 from alveolar macrophages ${ }^{146}$. Currently, two FDA-approved complement inhibitors are available, eculizumab and ravulizumab, both of which bind to $\mathrm{C} 5$ and sterically block the cleavage of C5 to C5a, and thus membrane attack complex formation. Four patients with COVID-19 receiving eculizumab intravenously showed a prompt reduction in C-reactive protein levels and an improvement of the lesions observed through chest computed tomography scan ${ }^{147}$. Among 35 patients admitted to the ICU with COVID-19, eculizumab treatment improved 15-day survival and hypoxia compared with patients treated with standard care alone $\mathrm{e}^{148}$. Trials with eculizumab (NCT04288713) and ravulizumab (NCT04369469, NCT04390464) are currently ongoing. In a report from Italy, a patient with severe COVID-19-related ARDS was safely and successfully treated with the compstatin-based complement C3 inhibitor AMY-101 (REF. ${ }^{149}$ ). A recent study comparing two different strategies targeting C3 and C5 has shown a general beneficial impact in patients with COVID-19, although C3 inhibition led to a broader therapeutic effect blocking cytokine dysregulation and NET generation, thereby having a positive impact on immunothrombosis-driven disease pathways ${ }^{150}$. The $\mathrm{C} 3$ inhibitor AMY-101 is currently being evaluated in three phase II clinical trials in patients with COVID-19-associated ARDS (EudraCT number 2020-001550-22, EudraCT number 2020-004408-32, NCT04395456). Another C3 inhibitor, the pegylated compound APL-9, is being tested in a phase I/II randomized controlled trial for severe COVID-19 (NCT04402060). The C1 
esterase inhibitor ruconest (NCT04414631, NCT04530136) and the C5a inhibitor IFX-1 (NCT04333420) are being tested as well.

\section{Conclusions}

Progressive advancements in our understanding of the pathophysiology of COVID-19 have been crucial for better management of COVID-19. Severe COVID-19 is not limited to the respiratory tract but is a multisystem syndrome in which the vascular endothelium is the most damaged organ, characterized by a state of immunothrombosis within microvessels (MicroCLOTS) and a predisposition to macrothrombosis. McGonagle et al. recently pointed out the potential role of thrombosis in pulmonary veins distal to the alveolar capillary bed, which should act as a clot filter, suggesting this could be a SARS-CoV-2related vasculitis responsible for ischaemic manifestations in different organs ${ }^{151}$. Based on all of these features, anticoagulant therapy and immunomodulatory agents are likely necessary to blunt the hyperinflammatory and prothrombotic conditions. Evidence from clinical trials is still limited and consensus-based guidelines recommend anticoagulation therapy based on an individual risk profile. With regard to immunomodulatory therapies, evidence is accumulating about a beneficial role in COVID-19. Timing of administration, however, is a challenging point that needs to be carefully considered, and data about a consistent effect on immunothrombosis are still lacking.

Aldo Bonaventura iD ${ }^{1,2,3 凶}$, Alessandra Vecchié ${ }^{1,3}$, Lorenzo Dagna ${ }^{4,5}$, Kimberly Martinod (iD) ${ }^{6}$, Dave L. Dixon ${ }^{1,7}$, Benjamin W. Van Tassell ${ }^{1,7}$, Francesco Dentali ${ }^{8}$, Fabrizio Montecucco (D) ${ }^{9,10}$, Steffen Massberg iD ${ }^{11,12}$, Marcel Levi ${ }^{13}$ and Antonio Abbate

${ }^{'}$ Pauley Heart Center, Division of Cardiology, Department of Internal Medicine, Virginia Commonwealth University, Richmond, VA, USA

${ }^{2}$ First Clinic of Internal Medicine, Department of Internal Medicine, University of Genoa, Genoa, Italy.

${ }^{3}$ Department of Internal Medicine, ASST dei Sette Laghi, Varese, Italy.

${ }^{4}$ Unit of Immunology, Rheumatology, Allergy and Rare Diseases, IRCCS San Raffaele Scientific Institute, Milan, Italy.

${ }^{5}$ Vita-Salute San Raffaele University, Milan, Italy.

${ }^{6}$ Center for Molecular and Vascular Biology, Department of Cardiovascular Sciences, KU Leuven, Leuven, Belgium.

${ }^{7}$ Department of Pharmacotherapy and Outcome Science, School of Pharmacy, Virginia Commonwealth University, Richmond, VA, USA.

${ }^{8}$ Department of Medicine and Surgery, Insubria University, Varese, Italy.

IRCCS Ospedale Policlinico San Martino Genova Italian Cardiovascular Network, Genoa, Italy.
${ }^{10}$ First Clinic of Internal Medicine, Department of Internal Medicine and Centre of Excellence for Biomedical Research (CEBR), University of Genoa, Genoa, Italy.

${ }^{1}$ Deutsches Zentrum für Herz-Kreislauf-Forschung (DZHK) e.V., Partner Site Munich Heart Alliance, Munich, Germany.

${ }^{12}$ Medizinische Klinik und Poliklinik I, Klinikum der Universität, Ludwig-Maximilians-Universität, Munich, Germany.

${ }^{13}$ Department of Medicine and Cardio-metabolic Programme - NIHR UCLH/UCL BRC, University College London Hospitals NHS Foundation Trust, London, UK.

凶e-mail:aldo.bonaventura@asst-settelaghi.it https://doi.org/10.1038/s41577-021-00536-9

Published online 6 April 2021

1. Pascarella, G. et al. COVID-19 diagnosis and management: a comprehensive review. J. Intern. Med 288, 192-206 (2020).

2. Bonaventura, A. et al. Targeting GM-CSF in COVID-19 pneumonia: rationale and strategies. Front. Immunol. 11, 1625 (2020)

3. McFadyen, J. D., Stevens, H. \& Peter, K. The emerging threat of (micro)thrombosis in COVID-19 and its therapeutic implications. Circ. Res. 127, 571-587 (2020).

4. Ranucci, M. et al. The procoagulant pattern of patients with COVID-19 acute respiratory distress syndrome. J. Thromb. Haemost. 18, 1747-1751 (2020).

5. Gupta, A. et al. Extrapulmonary manifestations of COVID-19. Nat. Med. 26, 1017-1032 (2020).

6. Fox, S. E. et al. Pulmonary and cardiac pathology in African American patients with COVID-19: an autopsy series from New Orleans. Lancet Respir. Med. 8 , 681-686 (2020).

7. Wichmann, D. et al. Autopsy findings and venous thromboembolism in patients with COVID-19: a prospective cohort study. Ann. Intern. Med. 173 268-277 (2020)

8. Carsana, L. et al. Pulmonary post-mortem finding in a series of COVID-19 cases from northern Italy: a two-centre descriptive study. Lancet Infect. Dis. 20, 1135-1140 (2020)

9. Schurink, B. et al. Viral presence and immunopathology in patients with lethal COVID-19: a prospective autopsy cohort study, Lancet Microbe 1, e290-e299 (2020).

10. Engelmann, B. \& Massberg, S. Thrombosis as an intravascular effector of innate immunity. Nat. Rev. Immunol. 13, 34-45 (2013).

11. Wiersinga, W. J., Rhodes, A., Cheng, A. C., Peacock, S. J. \& Prescott, H. C. Pathophysiology, transmission, diagnosis, and treatment of coronavirus disease 2019 (COVID-19): a review. JAMA 324, 782-793 (2020).

12. Berlin, D. A., Gulick, R. M. \& Martinez, F. J. Severe COVID-19. N. Engl. J. Med. 383, 2451-2460 (2020).

13. Lavezzo, $E$ et al Suppression of a SARS-CoV-2 outbreak in the Italian municipality of $\mathrm{Vo}^{\prime}$. Nature 584, 425-429 (2020).

14. Siddiqi, H. K. \& Mehra, M. R. COVID-19 illness in native and immunosuppressed states: a clinicaltherapeutic staging proposal. J. Heart Lung Transpl. 39, 405-407 (2020).

15. Buckley, L. F. et al. Role for anti-cytokine therapies in severe coronavirus disease 2019. Crit. Care Explor. 2 e0178 (2020).

16. Huang, C. et al. Clinical features of patients infected with 2019 novel coronavirus in Wuhan, China. Lancet 395, 497-506 (2020).

17. Vecchie, A. et al. IL-18 and infections: is there a role for targeted therapies? J. Cell Physiol. 236 1638-1657 (2021).

18. Fajgenbaum, D. C. \& June, C. H. Cytokine storm. N. Engl. J. Med. 383, 2255-2273 (2020)

19. Mehta, P. et al. COVID-19: consider cytokine storm syndromes and immunosuppression. Lancet 395 1033-1034 (2020).

20. Kox, M., Waalders, N. J. B., Kooistra, E. J., Gerretsen, J. $\varangle$ Pickkers, P. Cytokine levels in critically ill patients with COVID-19 and other conditions. JAMA 324 1565-1567 (2020)

21. Leisman, D. E. et al. Cytokine elevation in severe and critical COVID-19: a rapid systematic review, meta-analysis, and comparison with other inflammatory syndromes. Lancet Respir. Med. 8 , 1233-1244 (2020).
22. Blanco-Melo, D. et al. Imbalanced host response to SARS-CoV-2 drives development of COVID-19. Cell 181, 1036-1045.e9 (2020).

23. Toldo, S. et al. Inflammasome formation in the lungs of patients with fatal COVID-19. Inflamm. Res. 70, 7-10 (2021).

24. Rodrigues, T. S. et al. Inflammasomes are activated in response to SARS-CoV-2 infection and are associated with COVID-19 severity in patients. J. Exp. Med. 218 e20201707 (2021)

25. Quartuccio, L. et al. Profiling COVID-19 pneumonia progressing into the cytokine storm syndrome: results from a single Italian centre study on tocilizumab versus standard of care. J. Clin. Virol. 129, 104444 (2020).

26. Henry, B. M., de Oliveira, M. H. S., Benoit, S Plebani, M. \& Lippi, G. Hematologic, biochemical and immune biomarker abnormalities associated with severe illness and mortality in coronavirus disease 2019 (COVID-19): a meta-analysis. Clin. Chem. Lab. Med. 58, 1021-1028 (2020).

27. McGonagle, D., O’Donnell, J. S., Sharif, K., Emery, P. \& Bridgewood, C. Immune mechanisms of pulmonary intravascular coagulopathy in COVID-19 pneumonia. Lancet Rheumatol. 2, e437-e445 (2020).

28. Wang C et al. Alveolar macrophage dysfunction and cytokine storm in the pathogenesis of two severe COVID-19 patients. EBioMedicine 57, 102833 (2020).

29. Adachi, T. et al. Clinicopathologic and immunohistochemical findings from autopsy of patient with COVID-19, Japan. Emerg. Infect. Dis. 26 2157-2161 (2020).

30. Liao, M. et al. Single-cell landscape of bronchoalveola immune cells in patients with COVID-19. Nat. Med. 26, 842-844 (2020)

31. Alon, R. et al. Leukocyte trafficking to the lungs and beyond: lessons from influenza for COVID-19. Nat. Rev. Immunol. 21, 49-64 (2021).

32. Tay, M. Z., Poh, C. M., Renia, L., MacAry, P. A. $\& \mathrm{Ng}$, L. F. P. The trinity of COVID-19: immunity, inflammation and intervention. Nat. Rev. Immunol. 20, 363-374 (2020)

33. Tang, N., Li, D., Wang, X. \& Sun, Z. Abnormal coagulation parameters are associated with poor prognosis in patients with novel coronavirus pneumonia. J. Thromb. Haemost. 18, 844-847 (2020).

34. Panigada, M. et al. Hypercoagulability of COVID-19 patients in intensive care unit: a report of thromboelastography findings and other parameters of hemostasis. J. Thromb. Haemost. 18, 1738-1742 (2020).

35. Goshua, G. et al. Endotheliopathy in COVID-19 associated coagulopathy: evidence from a single-centre, cross-sectional study. Lancet Haematol. 7, e575-e582 (2020).

36. Iba, T., Levy, J. H., Levi, M., Connors, J. M. \& Thachil, J. Coagulopathy of coronavirus disease 2019 Crit. Care Med. 48, 1358-1364 (2020).

37. Lodigiani, C. et al. Venous and arterial thromboembolic complications in COVID-19 patients admitted to an academic hospital in Milan, Italy. Thromb. Res. 191, 9-14 (2020).

38. Iba, T., Connors, J. M. \& Levy, J. H. The coagulopathy, endotheliopathy, and vasculitis of COVID-19. Inflamm. Res. 69, 1181-1189 (2020).

39. Gazzaruso, C et al. Association between antithrombin and mortality in patients with COVID-19. A possible link with obesity. Nutr. Metab. Cardiovasc. Dis. 30 1914-1919 (2020).

40. Mir, N., D'Amico, A., Dasher, J., Tolwani, A. \& Valentine, V. Understanding the andromeda strain - the role of cytokine release, coagulopathy and antithrombin III in SARS-CoV2 critical illness. Blood Rev. 45, 100731 (2021).

41. Wang, Y. et al. SARS-CoV-2 infection of the live directly contributes to hepatic impairment in patients with COVID-19. J. Hepatol. 73, 807-816 (2020)

42. Portincasa, P., Krawczyk, M., Machill, A., Lammert, F. \& Di Ciaula, A. Hepatic consequences of COVID-19 infection. Lapping or biting? Eur. J. Intern. Med. 77 , 18-24 (2020)

43. Whyte, C. S., Morrow, G. B., Mitchell, J. L., Chowdary, P. \& Mutch, N. J. Fibrinolytic abnormalities in acute respiratory distress syndrome (ARDS) and versatility of thrombolytic drugs to treat COVID-19. J. Thromb. Haemost. 18, 1548-1555 (2020).

44. Gralinski, L. E. et al. Mechanisms of severe acute respiratory syndrome coronavirus-induced acute lung injury. mBio 4, e00271-13 (2013). 
45. Iba, T., Warkentin, T. E., Thachil, J., Levi, M. \& Levy, J. H. Proposal of the definition for COVID-19-associated coagulopathy. J. Clin. Med. 10, 191 (2021).

46. Tian, S. et al. Pulmonary pathology of early-phase 2019 novel coronavirus (COVID-19) pneumonia in two patients with lung cancer. J. Thorac. Oncol. 15, 700-704 (2020)

47. Varga, Z. et al. Endothelial cell infection and endotheliitis in COVID-19. Lancet 395, 1417-1418 (2020).

48. Schaller, T. et al. Postmortem examination of patients with COVID-19. JAMA 323, 2518-2520 (2020).

49. Nicholls, J. M. et al. Lung pathology of fatal severe acute respiratory syndrome. Lancet 361, 1773-1778 (2003).

50. McGonagle, D., Sharif, K., O'Regan, A. \& Bridgewood, $C$. The role of cytokines including interleukin- 6 in COVID-19 induced pneumonia and macrophage activation syndrome-like disease. Autoimmun. Rev. 19, 102537 (2020).

51. Chen, L. \& Hao, G. The role of angiotensin-converting enzyme 2 in coronaviruses/influenza viruses and cardiovascular disease. Cardiovasc. Res. 116 1932-1936 (2020)

52. Magro, C. et al. Complement associated microvascular injury and thrombosis in the pathogenesis of severe COVID-19 infection: a report of five cases. Trans/ Res. 220, 1-13 (2020)

53. Zhang, H. et al. Histopathologic changes and SARS-CoV-2 immunostaining in the lung of a patient with COVID-19. Ann. Intern. Med. 172, 629-632 (2020).

54. Xu, Z. et al. Pathological findings of COVID-19 associated with acute respiratory distress syndrome. Lancet Respir. Med. 8, 420-422 (2020).

55. Teuwen, L. A., Geldhof, V., Pasut, A. \& Carmeliet, P. COVID-19: the vasculature unleashed. Nat. Rev. Immunol. 20, 389-391 (2020).

56. Goeijenbier, M. et al. Review: Viral infections and mechanisms of thrombosis and bleeding. J. Med. Virol. 84, 1680-1696 (2012).

57. Guo, Y Korteweg C. McNutt, M. A \& Gu, J. Pathogenetic mechanisms of severe acute respiratory syndrome. Virus Res. 133, 4-12 (2008).

58. Ding, Y. et al. The clinical pathology of severe acute respiratory syndrome (SARS): a report from China. J. Pathol. 200, 282-289 (2003).

59. Hamming, I. et al. Tissue distribution of ACE2 protein, the functional receptor for SARS coronavirus. A first step in understanding SARS pathogenesis. J. Pathol. 203, 631-637 (2004).

60. Levi, M. \& van der Poll, T. Coagulation and sepsis. Thromb. Res. 149, 38-44 (2017)

61. Helms, J. et al. High risk of thrombosis in patient with severe SARS-CoV-2 infection: a multicenter prospective cohort study. Intensive Care Med. $\mathbf{4 6}$ 1089-1098 (2020).

62. Escher, R., Breakey, N. \& Lammle, B. Severe COVID-19 infection associated with endothelial activation. Thromb. Res. 190, 62 (2020).

63. Martinelli, N. et al. A relative ADAMTS13 deficiency supports the presence of a secondary microangiopath in COVID 19. Thromb. Res. 193, 170-172 (2020).

64. Schwameis, M., Schorgenhofer, C., Assinger, A. Steiner, M. M. \& Jilma, B. VWF excess and ADAMTS13 deficiency: a unifying pathomechanism linking inflammation to thrombosis in DIC, malaria, and TTP. Thromb. Haemost 113, 708-718 (2015).

65. Guervilly, C. et al. Circulating endothelial cells as a marker of endothelial injury in severe COVID-19. J. Infect. Dis. 222, 1789-1793 (2020).

66. Falcinelli, E. et al. Role of endothelial dysfunction in the thrombotic complications of COVID-19 patients. J. Infect. https://doi.org/10.1016/j.jinf.2020.11.041 (2020).

67. Marini, J. J. \& Gattinoni, L. Management of COVID-19 respiratory distress. JAMA 323, 2329-2330 (2020).

68. Perico, L. et al. Immunity, endothelial injury and complement-induced coagulopathy in COVID-19. Nat. Rev. Nephrol. 17, 46-64 (2021).

69. Bonetti, P. O., Lerman, L. O. \& Lerman, A. Endothelial dysfunction: a marker of atherosclerotic risk. Arterioscler. Thromb. Vasc. Biol. 23, 168-175 (2003).

70. Hottz, E. D. et al. Platelet activation and plateletmonocyte aggregate formation trigger tissue factor expression in patients with severe COVID-19. Blood 136, 1330-1341 (2020).

71. Nicolai, L. et al. Immunothrombotic dysregulation in COVID-19 pneumonia is associated with respiratory failure and coagulopathy. Circulation 142, 1176-1189 (2020).
72. Levi, M. Platelets in critical illness. Semin. Thromb. Hemost. 42, 252-257 (2016).

73. Zaid, Y. et al. Platelets can associate with SARS-Cov-2 RNA and are hyperactivated in COVID-19. Circ. Res. 127, 1404-1418 (2020)

74. Chrysanthopoulou, A. et al. Interferon $\lambda 1 /$ IL-29 and inorganic polyphosphate are novel regulators of neutrophil-driven thromboinflammation. J. Pathol. 243, 111-122 (2017).

75. Gu, S. X. et al. Thrombocytopathy and endotheliopathy: crucial contributors to COVID-19 thromboinflammation. Nat. Rev. Cardiol. 18 194-209 (2021)

76. Gattinoni, L., Chiumello, D. \& Rossi, S. COVID-19 pneumonia: ARDS or not? Crit. Care 24, 154 (2020)

77. Gattinoni, L. et al. COVID-19 does not lead to a 'typical' acute respiratory distress syndrome. Am. J. Respir. Crit. Care Med. 201, 1299-1300 (2020).

78. Gattinoni, L. et al. COVID-19 pneumonia: different respiratory treatments for different phenotypes? Intensive Care Med. 46, 1099-1102 (2020).

79. Ciceri, F. et al. Microvascular COVID-19 lung vessels obstructive thromboinflammatory syndrome (MicroCLOTS): an atypical acute respiratory distress syndrome working hypothesis. Crit. Care Resusc. 22, 95-97 (2020)

80. Middleton, E. A. et al. Neutrophil extracellular traps contribute to immunothrombosis in COVID-19 acute respiratory distress syndrome. Blood 136 , 1169-1179 (2020)

81. Mitsios, A., Arampatzioglou, A., Arelaki, S Mitroulis, I. \& Ritis, K. NETopathies? Unraveling the dark side of old diseases through neutrophils Front. Immunol. 7, 678 (2016).

82. Rebetz, J., Semple, J. W. \& Kapur, R. The pathogenic involvement of neutrophils in acute respiratory distress syndrome and transfusion-related acute lung injury. Transfus. Med. Hemother 45, 290-298 (2018).

83. Bendib, I. et al. Neutrophil extracellular traps are elevated in patients with pneumonia-related acute respiratory distress syndrome. Anesthesiology 130 581-591 (2019)

84. Lv, X. et al. Extracellular histones are clinically relevant mediators in the pathogenesis of acute respiratory distress syndrome. Respir. Res. 18, 165 (2017).

85. Zuo, Y. et al. Neutrophil extracellular traps in COVID-19. JCI Insight 5, e138999 (2020).

86. Skendros, P. et al. Complement and tissue factor-enriched neutrophil extracellular traps are key drivers in COVID-19 immunothrombosis. J. Clin Invest. 130, 6151-6157 (2020)

87. Leppkes, M. et al. Vascular occlusion by neutrophil extracellular traps in COVID-19. EBioMedicine 58 , 102925 (2020).

88. Radermecker, C. et al. Neutrophil extracellular traps infiltrate the lung airway, interstitial, and vascular compartments in severe COVID-19. J. Exp. Med. 217 e20201012 (2020)

89. Veras, F. P. et al. SARS-CoV-2-triggered neutrophil extracellular traps mediate COVID-19 pathology. J. Exp. Med. 217, e20201129 (2020)

90. O’Flynn, J., Dixon, K. O., Faber Krol, M. C. Daha, M. R. \& van Kooten, C. Myeloperoxidase directs properdin-mediated complement activation. J. Innate Immun 6, 417-425 (2014).

91. de Bont, C. M., Boelens, W. C. \& Pruijn, G. J. M. NETosis, complement, and coagulation: a triangular relationship. Cell Mol. Immunol. 16, 19-27 (2019).

92. Yalavarthi, S. et al. Release of neutrophil extracellular traps by neutrophils stimulated with antiphospholipid antibodies: a newly identified mechanism of thrombosis in the antiphospholipid syndrom Arthritis Rheumatol. 67, 2990-3003 (2015)

93. Mendoza-Pinto, C. Garcia-Carrasco, M. \& Cervera, R. Role of infectious diseases in the antiphospholipid syndrome (including its catastrophic variant). Curr. Rheumatol. Rep. 20, 62 (2018).

94. Zuo, Y. et al. Prothrombotic autoantibodies in serum from patients hospitalized with COVID-19. Sci. TransI Med. 12, eabd3876 (2020).

95. Channappanavar, R. \& Perlman, S. Pathogenic human coronavirus infections: causes and consequences of cytokine storm and immunopathology. Semin. Immunopathol. 39, 529-539 (2017).

96. Chousterman, B. G. Swirski, F. K. \& Weber, G. F. Cytokine storm and sepsis disease pathogenesis. Semin. Immunopathol. 39, 517-528 (2017).

97. Bosmuller, $\mathrm{H}$. et al. The evolution of pulmonary pathology in fatal COVID-19 disease: an autopsy study with clinical correlation. Virchows Arch. 477 349-357 (2020).

98. Wolfel, R. et al. Virological assessment of hospitalized patients with COVID-2019. Nature 581, 465-469 (2020).

99. Bradley, B. T. et al. Histopathology and ultrastructural findings of fatal COVID-19 infections in Washington State: a case series. Lancet 396, 320-332 (2020)

100. Manne, B. K. et al. Platelet gene expression and function in patients with COVID-19. Blood 136 , 1317-1329 (2020).

101. Zhang, S. et al. SARS-CoV-2 binds platelet ACE2 to enhance thrombosis in COVID-19. J. Hematol. Oncol. 13, 120 (2020).

102. Yan, S. F., Mackman, N., Kisiel, W., Stern, D. M. \& Pinsky, D. J. Hypoxia/hypoxemia-induced activation of the procoagulant pathways and the pathogenesis of ischemia-associated thrombosis. Arterioscler. Thromb. Vasc. Biol. 19, 2029-2035 (1999).

103. Bikdeli, B. et al. COVID-19 and thrombotic or thromboembolic disease: implications for prevention, antithrombotic therapy, and follow-up: JACC State-of-the-Art Review. J. Am. Coll. Cardiol. 75, 2950-2973 (2020).

104. Wang, T. et al. Attention should be paid to venous thromboembolism prophylaxis in the management of COVID-19. Lancet Haematol. 7, e362-e363 (2020).

105. Paranjpe, I. et al. Association of treatment dose anticoagulation with in-hospital survival among hospitalized patients with COVID-19. J. Am. Coll. Cardiol. 76, 122-124 (2020).

106. Barnes, G. D. et al. Thromboembolism and anticoagulant therapy during the COVID-19 pandemic: interim clinical guidance from the anticoagulation forum. J. Thromb. Thrombolysis 50, 72-81 (2020).

107. Levi, M. \& Hunt, B. J. Thrombosis and coagulopathy in COVID-19: an illustrated review. Res. Pract. Thromb. Haemost. 4, 744-751 (2020).

108. Paar, V. et al. Anti-coagulation for COVID-19 treatment both anti-thrombotic and anti-inflammatory? J. Thromb. Thrombolysis 51, 226-231 (2021).

109. Prandoni, P. et al. The hazard of fondaparinux in non-critically ill patients with COVID-19: retrospective controlled study versus enoxaparin. Thromb. Res. 196, 395-397 (2020)

110. Russo, V. et al. Fondaparinux use in patients with COVID-19: a preliminary multicenter real-world experience. J. Cardiovasc. Pharmacol. 76, 369-371 (2020).

111. Rossi, R., Coppi, F., Talarico, M. \& Boriani, G. Protective role of chronic treatment with direct oral anticoagulants in elderly patients affected by interstitial pneumonia in COVID-19 era. Eur. J. Intern. Med. 77, 158-160 (2020).

112. Flam, B., Wintzell, V., Ludvigsson, J. F., Martensson, J. $\&$ Pasternak, B. Direct oral anticoagulant use and risk of severe COVID-19. J. Intern. Med. 289, 411-419 (2021).

113. Larsson, M. et al. A factor XIla inhibitory antibody provides thromboprotection in extracorporeal circulation without increasing bleeding risk. Sci. Trans/ Med. 6, $222 \mathrm{ra} 217$ (2014).

114. Jansen, P. M. et al. Inhibition of factor XII in septic baboons attenuates the activation of complement and fibrinolytic systems and reduces the release of interleukin-6 and neutrophil elastase. Blood 87. 2337-2344 (1996).

115. Asakura, H. \& Ogawa, H. Potential of heparin and nafamostat combination therapy for COVID-19. J. Thromb. Haemost. 18, 1521-1522 (2020).

116. Yamamoto, M. et al. Identification of nafamostat as a potent inhibitor of Middle East respiratory syndrome coronavirus $\mathrm{S}$ protein-mediated membrane fusion using the split-protein-based cell-cell fusion assay. Antimicrob. Agents Chemother. 60, 6532-6539 (2016).

117. Bonaventura, A., Montecucco, F. \& Dallegri, F. Update on the effects of treatment with recombinant tissue-type plasminogen activator (rt-PA) in acute ischemic stroke. Expert. Opin. Biol. Ther. 16, 1323-1340 (2016).

118. Liu, C. et al. Meta-analysis of preclinical studies of fibrinolytic therapy for acute lung injury. Front. Immunol. 9, 1898 (2018).

119. Wang, J. et al. Tissue plasminogen activator (tPA) treatment for COVID-19 associated acute respiratory distress syndrome (ARDS): a case series. J. Thromb. Haemost. 18, 1752-1755 (2020).

120. Kanthi, Y., Knight, J. S., Zuo, Y. \& Pinsky, D. J. New (re) purpose for an old drug: purinergic modulation may extinguish the COVID-19 thromboinflammatory firestorm. JCl Insight 5, e 140971 (2020). 
121. Fata-Hartley, C. L. \& Palmenberg, A. C. Dipyridamole reversibly inhibits mengovirus RNA replication. J. Virol. 79, 11062-11070 (2005).

122. Huang, B. et al. Mucosal profiling of pediatric-onset colitis and IBD reveals common pathogenics and therapeutic pathways. Cell 179, 1160-1176.e24 (2019).

123. Insel, P. A. et al. cAMP and Epac in the regulation of tissue fibrosis. Br. J. Pharmacol. 166, 447-456 (2012).

124. Ali, R. A. et al. Adenosine receptor agonism protects against NETosis and thrombosis in antiphospholipid syndrome. Nat. Commun. 10, 1916 (2019).

125. Liu, X. et al. Potential therapeutic effects of dipyridamole in the severely ill patients with COVID-19. Acta Pharm. Sin. B 10, 1205-1215 (2020).

126. Mitsios, A. et al. Ticagrelor exerts immune-modulatory effect by attenuating neutrophil extracellular traps. Int. J. Mol. Sci. 21, 3625 (2020)

127. Stebbing, J. et al. JAK inhibition reduces SARS-CoV-2 liver infectivity and modulates inflammatory responses to reduce morbidity and mortality. Sci. Adv. 7 eabe4724 (2020).

128. Cavalli, G. et al. Repurposing of biologic and targeted synthetic anti-rheumatic drugs in COVID-19 and hyper-inflammation: a comprehensive review of available and emerging evidence at the peak of the pandemic. Front. Pharmacol. 11, 598308 (2020).

129. Barnes, B. J. et al. Targeting potential drivers of COVID-19: neutrophil extracellular traps. J. Exp. Med. 217, e20200652 (2020).

130. Mohamed, M. M. A. El-Shimy, I. A. \& Hadi, M. A. Neutrophil elastase inhibitors: a potential prophylactic treatment option for SARS-CoV-2-induced respiratory complications? Crit. Care 24, 311 (2020).

131. Zeiher, B. G. et al. Neutrophil elastase inhibition in acute lung injury: results of the STRIVE study. Crit. Care Med. 32, 1695-1702 (2004).

132. Aikawa, N. \& Kawasaki, Y. Clinical utility of the neutrophil elastase inhibitor sivelestat for the treatment of acute respiratory distress syndrome. Ther. Clin. Risk Manag. 10, 621-629 (2014).

133. Tagami, T. et al. Effect of a selective neutrophil elastase inhibitor on mortality and ventilator-free days in patients with increased extravascular lung water: a post hoc analysis of the PiCCO Pulmonary Edema Study. J. Intensive Care 2, 67 (2014).

134. Papayannopoulos, V., Staab, D. \& Zychlinsky, A. Neutrophil elastase enhances sputum solubilization in cystic fibrosis patients receiving DNase therapy. PLOS ONE 6, e28526 (2011).

135. Zou, Y. et al. Neutrophil extracellular traps promote lipopolysaccharide-induced airway inflammation and mucus hypersecretion in mice. Oncotarget 9 , 13276-13286 (2018).

136. Lefrancais, E., Mallavia, B., Zhuo, H., Calfee, C. S. $\&$ Looney, M. R. Maladaptive role of neutrophil extracellular traps in pathogen-induced lung injury. JCI Insight 3, e98178 (2018).

137. Thomas, G. M. et al. Extracellular DNA traps are associated with the pathogenesis of TRALI in humans and mice. Blood 119, 6335-6343 (2012).

138. Okur, H. K. et al. Preliminary report of in vitro and in vivo effectiveness of dornase alfa on SARS-CoV- 2 infection. N. Microbes N. Infect. 37, 100756 (2020).

139. Weber, A. G., Chau, A. S., Egeblad, M., Barnes, B. J. \& Janowitz, T. Nebulized in-line endotracheal dornase alfa and albuterol administered to mechanically ventilated COVID-19 patients: a case series. Mol. Med. 26, 91 (2020).

140. Gollomp, K. et al. Neutrophil accumulation and NET release contribute to thrombosis in HIT. JCI Insight 3 . e99445 (2018)

141. Fuchs, T. A. et al. Extracellular DNA traps promote thrombosis. Proc. Natl Acad. Sci. USA 107 15880-15885 (2010).

142. Wolach, O. et al. Increased neutrophil extracellular trap formation promotes thrombosis in myeloproliferative neoplasms. Sci. Transl Med. 10 eaan8292 (2018)

143. Yost, C. C. et al. Neonatal NET-inhibitory factor and related peptides inhibit neutrophil extracellular trap formation. J Clin Invest 126, 3783-3798 (2016).

144. Java, A. et al. The complement system in COVID-19: friend and foe? JCI Insight 5, e 140711 (2020).

145. Zhang, Y. et al. Coagulopathy and antiphospholipid antibodies in patients with COVID-19. N. Engl. J. Med. 382, e38 (2020).

146. Risitano, A. M. et al. Complement as a target in COVID-19? Nat. Rev. Immunol. 20, 343-344 (2020).
147. Diurno, F. et al. Eculizumab treatment in patients with COVID-19: preliminary results from real life ASL Napoli 2 Nord experience. Eur. Rev. Med. Pharmacol. Sci. 24, 4040-4047 (2020)

148. Annane, D. et al. Eculizumab as an emergency treatment for adult patients with severe COVID-19 in the intensive care unit: a proof-of-concept study. EClinicalMedicine 28, 100590 (2020).

149. Mastaglio, S. et al. The first case of COVID-19 treated with the complement C3 inhibitor AMY-101 Clin. Immunol. 215, 108450 (2020).

150. Mastellos, D. C. et al. Complement C3 vs C5 inhibition in severe COVID-19: early clinical findings reveal differential biological efficacy. Clin. Immunol. 220 108598 (2020).

151. McGonagle, D., Bridgewood, C., Ramanan, A. V. Meaney, J. F. M. \& Watad, A. COVID-19 vasculitis and novel vasculitis mimics. Lancet Rheumatol. 3 , e224-e233 (2021)

152. Tang, N. et al. Anticoagulant treatment is associated with decreased mortality in severe coronavirus disease 2019 patients with coagulopathy. J. Thromb. Haemost 18, 1094-1099 (2020).

153. Ayerbe, L., Risco, C. \& Ayis, S. The association between treatment with heparin and survival in patients with COVID-19. J. Thromb. Thrombolysis 50 298-301 (2020)

154. Nadkarni, G. N. et al. Anticoagulation, bleeding, mortality, and pathology in hospitalized patients with COVID-19. J. Am. Coll. Cardiol. 76, 1815-1826 (2020).

155. La Rosee, F. et al. The Janus kinase $1 / 2$ inhibitor ruxolitinib in COVID-19 with severe systemic hyperinflammation. Leukemia 34, 1805-1815 (2020).

156. Cao, Y. et al. Ruxolitinib in treatment of severe coronavirus disease 2019 (COVID-19): a multicenter, single-blind, randomized controlled trial. J. Allergy Clin. Immunol. 146, 137-146.e3 (2020).

157. Hickey, M. J. \& Kubes, P. Intravascular immunity: the host-pathogen encounter in blood vessels. Nat. Rev. Immunol. 9, 364-375 (2009)

158. Jackson, S. P., Darbousset, R. \& Schoenwaelder, S. M Thromboinflammation: challenges of therapeutically targeting coagulation and other host defense mechanisms. Blood 133, 906-918 (2019).

159. Massberg, S. et al. Reciprocal coupling of coagulation and innate immunity via neutrophil serine proteases. Nat. Med. 16, 887-896 (2010).

160. von Bruhl, M. L. et al. Monocytes, neutrophils, and platelets cooperate to initiate and propagate venous thrombosis in mice in vivo. J. Exp. Med. 209 819-835 (2012).

161. Kambas, K. et al. Autophagy mediates the delivery of thrombogenic tissue factor to neutrophil extracellular traps in human sepsis. PLOS ONE 7, e45427 (2012).

162. Bonaventura, A. et al. The pathophysiological role of neutrophil extracellular traps in inflammatory diseases. Thromb. Haemost. 118, 6-27 (2018).

163. Martinod, K. $\&$ Wagner, D. D. Thrombosis: tangled up in NETs. Blood 123, 2768-2776 (2014).

164. Ammollo, C. T., Semeraro, F., Xu, J., Esmon, N. L. \& Esmon, C. T. Extracellular histones increase plasma thrombin generation by impairing thrombomodulindependent protein C activation. J. Thromb. Haemost. 9, 1795-1803 (2011).

165. Nicolai, L., Gaertner, F. \& Massberg, S. Platelets in host defense: experimental and clinical insights. Trends Immunol. 40, 922-938 (2019).

166. Ma, A. C. \& Kubes, P. Platelets, neutrophils, and neutrophil extracellular traps (NETs) in sepsis. J. Thromb. Haemost. 6, 415-420 (2008).

167. Guo, L. \& Rondina, M. T. The era of thromboinflammation: platelets are dynamic sensors and effector cells during infectious diseases. Front Immunol 10, 2204 (2019).

168. Reinhardt, C. et al. Protein disulfide isomerase acts as an injury response signal that enhances fibrin generation via tissue factor activation. J. Clin. Invest. 118, 1110-1122 (2008).

169. Semple, J. W., Aslam, R., Kim, M., Speck, E. R. \& Freedman, J. Platelet-bound lipopolysaccharide enhances Fc receptor-mediated phagocytosis of IgG-opsonized platelets. Blood 109, 4803-4805 (2007).

170. Zhang, G. et al. Lipopolysaccharide stimulates platelet secretion and potentiates platelet aggregation via TLR4/MyD88 and the cGMP-dependent protein kinase pathway. J. Immunol. 182, 7997-8004 (2009)

171. Bergmann, S. \& Hammerschmidt, S. Fibrinolysis and host response in bacterial infections. Thromb Haemost. 98, 512-520 (2007).

172. Buchanan, J. T. et al. DNase expression allows the pathogen group A Streptococcus to escape killing in neutrophil extracellular traps. Curr. Biol. 16 396-400 (2006).

173. Beiter, K. et al. An endonuclease allows Streptococcus pneumoniae to escape from neutrophil extracellular traps. Curr. Biol. 16, 401-407 (2006).

174. Cheng, A. G. et al. Contribution of coagulases towards Staphylococcus aureus disease and protective immunity. PLoS Pathog. 6, e1001036 (2010).

175. Guggenberger, C., Wolz, C., Morrissey, J. A. \& Heesemann, J. Two distinct coagulase-dependent barriers protect Staphylococcus aureus from neutrophils in a three dimensional in vitro infection model. PLoS Pathog. 8, e1002434 (2012).

176. Gavriilaki, E. \& Brodsky, R. A. Complementopathies and precision medicine. J. Clin. Invest. 130 , 2152-2163 (2020).

177. Gralinski, L. E. et al. Complement activation contributes to severe acute respiratory syndrome coronavirus pathogenesis. mBio 9, e01753-18 (2018).

178. Markiewski, M. M., Nilsson, B., Ekdahl, K. N., Mollnes, T. E. \& Lambris, J. D. Complement and coagulation: strangers or partners in crime? Trends Immunol. 28, 184-192 (2007).

179. Ramlall, V. et al. Immune complement and coagulation dysfunction in adverse outcomes of SARS-CoV-2 infection. Nat. Med. 26, 1609-1615 (2020).

180. Peerschke, E. I., Reid, K. B. \& Ghebrehiwet, B. Platelet activation by $\mathrm{C} 1 \mathrm{q}$ results in the induction of $\alpha \mathrm{llb} / \beta 3$ integrins (GPIIb-IIla) and the expression of P-selectin and procoagulant activity. J. Exp. Med 178, 579-587 (1993)

181. Ikeda, K. et al. C5a induces tissue factor activity on endothelial cells. Thromb. Haemost. 77, 394-398 (1997).

182. Ritis, K. et al. A novel C5a receptor-tissue factor cross-talk in neutrophils links innate immunity to coagulation pathways. J. Immunol. 177, 4794-4802 (2006).

Acknowledgements

F.M. is supported by a grant from the Italian Ministry of Health to the Italian Cardiovascular Network (\#2754291).

\section{Author contributions}

A.B., A.V., K.M., S.M., M.L. and A.A. contributed to all aspects of the article. L.D., D.L.D., B.W.V.T., F.D. and F.M contributed to discussion of the content and reviewed and/or edited the manuscript before submission. All authors approved the final version of the manuscript.

\section{Competing interests}

A.B. and A.V. received a travel grant from Kiniksa Pharmaceuticals Ltd and honoraria from Effetti s.r.l. (Milan, Italy). L.D. has received consultation honoraria from Abbvie, Amgen, Biogen, Bristol-Myers Squibb, Celltrion, GlaxoSmithKline, Novartis, Pfizer, Roche, Sanofi-Genzyme and SOBI. K.M. is an inventor on patent US9642822 and patent applications WO20180271953A1 and US2019167680A1, and is a consultant for PEEL Therapeutics, Inc. B.W.V.T has served as a consultant for Novartis and Serpin Pharma, and has received research support from Novartis, Kiniksa Pharmaceuticals Ltd, Serpin Pharma and SOBI. A.A. has served as a consultant for Applied Clinical Intel, AstraZeneca, Cromos Pharma, Janssen, Kiniksa Pharmaceuticals Ltd, Merck, Olatec and Serpin Pharma, and has received research support from Janssen, Olatec and Serpin Pharma. All other authors declare no competing interests.

Peer review information

Nature Reviews Immunology thanks the anonymous reviewers for their contribution to the peer review of this work.

\section{Publisher's note}

Springer Nature remains neutral with regard to jurisdictional claims in published maps and institutional affiliations.

Supplementary information

The online version contains supplementary material available at https://doi.org/10.1038/s41577-021-00536-9.

(C) Springer Nature Limited 2021 